\title{
Workforce planning incorporating skills: State of the art
}

\author{
Philippe De Bruecker* \\ KU Leuven, Research Center for Operations Management, Leuven (Belgium) \\ Jorne Van den Bergh* \\ HUBrussel, Center for Informatics, Modeling and Simulation, Brussels (Belgium) \\ Jeroen Beliën* \\ HUBrussel, Center for Informatics, Modeling and Simulation, Brussels (Belgium) \\ Erik Demeulemeester* \\ KU Leuven, Research Center for Operations Management, Leuven (Belgium)
}

\begin{abstract}
This paper presents a review and classification of the literature regarding workforce planning problems incorporating skills. In many cases, technical research regarding workforce planning focuses very hard on the mathematical model and neglects the real life implications of the simplifications that were needed for the model to perform well. On the other hand, many managerial studies give an extensive description of the human implications of certain management decisions in particular cases, but fail to provide useful mathematical models to solve workforce planning problems. This review will guide the operations researcher in his search to find useful papers and information regarding workforce planning problems incorporating skills. We not only discuss the differences and similarities between different papers, but we also give an overview of the managerial insights. The objective is to present a combination of technical and managerial knowledge to encourage the production of more realistic and useful solution techniques.
\end{abstract}

Keywords: Skills, workforce staffing and scheduling, literature review.

\footnotetext{
${ }^{*}$ Corresponding author

Email addresses: philippe.debruecker@kuleuven.be (Philippe De Bruecker ), jorne.vandenbergh@kuleuven. be (Jorne Van den Bergh), jeroen.belien@kuleuven.be (Jeroen Beliën), erik.demeulemeester@kuleuven.be (Erik Demeulemeester)
} 


\section{Introduction}

The planning of the workforce in a company is one of the most difficult problems managers face. As the size of the company increases, the problem tends to get more and more difficult. The workforce planning defines when and how many employees should be hired or dismissed and when these employees should work. Hence, it is a combination of staffing and scheduling decisions. Workforce planning problems entail some special features that are absent in all other types of resource allocation problems. When people are involved, the decision environment tends to get very dynamic and workforce planners face an extremely heterogeneous set of employees. One must not only take into account different employee preferences and union constraints, but also the different skills that workers may possess.

In this research, we define skills as the ability of a worker to perform certain tasks well. In certain problems, some tasks can only be carried out by personnel members who possess a specific skill and/or skill level. Other problems focus on the differences in performance of differently skilled persons and try to maximize some company specific performance measures like quality or speed of work. Sometimes, differently skilled persons entail different costs and the company wants to minimize the total labor costs. In these examples, the skills of a worker have an intuitive and direct effect on task restrictions, efficiency, quality or costs. However, incorporating skills in a workforce planning problem often involves some unexpected effects too that are not always easy to grasp correctly. Skill substitution and cross-training, for example, can have a positive or negative effect on performance depending on the situation. In addition, incorporating skills also entails learning effects, the effects of mixing different types of skills and the effects of teamwork.

On top of these complex consequences of different skills, finding a good solution technique appears to be even more difficult, especially when real-life problems are considered.

\section{Research objectives}

The objective of this paper is to provide an overview and classification of the literature to guide the operations researcher in his search to find useful papers and information regarding workforce planning problems incorporating skills. The unique feature of this approach is that we not only consider the relevant technical literature, but also the managerial literature. The main idea is that skill related workforce decisions actually belong to human relations management, but should be made based on sound technical methodologies. Unfortunately, only few papers succeed in combining both aspects. On the one hand, technical research regarding workforce planning usually focuses on the mathematical model and neglects the real life implications of the simplifications that were needed for the model to perform well. The inclusion of skills into the problem remains in many cases limited to the section of future research. Another problem that arises is that the formulated model is not yet applied or tested

with real data in many papers [111]. On the other hand, many managerial studies 
give an extensive description of the human implications of certain management decisions in particular cases, but fail to provide useful mathematical models to solve the problem [30].

This paper first focuses on the managerial knowledge regarding skills in workforce planning problems. Next, the state of the art of the technical knowledge in operations management literature is reviewed concerning mathematical models for solving workforce planning problems incorporating skills. Many technical research papers do not consider all possible consequences of certain decisions regarding skills or make wrong or incomplete assumptions in their model. Such models do not represent a realistic scenario and are likely to fail in real life applications. In Section 3, we investigate the dynamics of different skill types that are used in the literature to obtain a good representation of reality. This is useful for the operations researcher to check the correctness and completeness of his model. In Section 4, an overview is presented of the techniques that are used to model and solve different skill related workforce planning problems.

To search for relevant literature on skill related workforce planning, we first looked for review articles. Unfortunately, no review papers exist that are dedicated to skills in workforce planning problems. Some review papers exist that focus on general workforce planning problems $[5,31,53,81,111]$, but none go into detail regarding skills. The collection of articles reviewed in this research consists of the relevant articles presented by the previous general review papers $[5,31,53,81,111]$ and the articles resulting from our direct search as well as references therein. We limited our search to papers published after the year 2004 and the cut-off date is December 2012. Exceptions were made for interesting older papers that were cited multiple times.

To the best of our knowledge, this paper is the first literature review that combines managerial insights and technical and mathematical knowledge to guide the operations researchers to solve workforce planning problems incorporating skills.

\section{Managerial aspects of skill related workforce planning}

As an operations researcher, it is important to identify all potential factors that may impact the workforce decisions. In this section, different types of skills are defined and we analyze the impact of different skill types on the problem formulation.

\subsection{Definition of skills}

In this literature review we define skills as the ability to perform certain tasks well. As this is a very broad definition, we identify two different skill classes to structure our analysis of the skill literature; the hierarchical class and the categorical class. Next, we consider six main skill determinants that are used in the literature to determine skills. Finally, we look at the performance measures used in operations research papers that are impacted by the skill level of the employees.

Defining different classes and types of skills facilitates the investigation of the different consequences and dynamics of including skills in a workforce planning problem. This is very helpful since many researchers, e.g., Buchan and Dal Poz [30], Markes [94] and Gibbs et al. [61] complain about the haziness concerning the effects of skill 
decisions. Markes [94] also mentions that there still remains some uncertainty concerning the impact of different ways to manage the skill pool. This section gives an overview of the dynamics and effects of skills presented in the literature in order to reduce the haziness surrounding skills.

\subsubsection{Skill classes}

By analyzing the technical and managerial literature concerning skills in workforce planning, we discovered two different skill classes: the hierarchical class and the categorical class. This classification is very useful because both classes appear to have different effects when skill substitution and cross-training is allowed (see Section 3.2).

In case of hierarchical skills, workers with a lower skill level can do less than workers with a higher skill level. Workers with a higher skill level are more educated or have more experience and can therefore perform more tasks, or they can perform certain tasks better or faster. When skills have a hierarchical nature, higher skilled persons can perform the tasks that are normally performed by a lesser skilled person. This is referred to as substitution. While substitution is always possible in this case, the company can decide whether or not to allow it. Hierarchical skills are usually defined on a continuous scale but are sometimes discretized in several skill levels. Some researchers even only use two levels: skilled or unskilled [42, 58, 65, 83, 84, 106].

In case of categorical skills, there is no difference in skill level and the skills of a worker determine which tasks he or she can perform. In this case, the skills of one person are not better or worse than the skills of another person. Hence, the different skills cannot be hierarchically ranked. When a person has a skill that allows him to perform more than one task, he actually possesses all separate skills to perform each single task. We refer to a worker who possesses different categorical skills as a cross-trained worker.

Table 1 shows the papers that make use of hierarchical and categorical skills. While most papers only consider one skill class in their problem, some researchers consider both classes at the same time $[19,24,51,52,56,57,70,110]$ and talk about skill domains or categories and skill levels. In these papers, the authors assume that there exists a hierarchical structure in each skill category. Eiselt and Marianov [51], for example, position each employee and each task in a skill space. Each dimension in this space represents a different skill and the position in each dimension represents the skill level. As is discussed in Section 3.2, different skill classes can have different implications. Therefore, the definition of the considered skill class is very important since it determines which dynamics will come into play. Fortunately, the number of papers that do not define the skill class is very limited [15, 49, 55, 66, 76]. 
Table 1: Overview of the literature using hierarchical and categorical skill types

\begin{tabular}{ll}
\hline Hierarchical task skills & {$[1,2,3,9,10,11,12,16,17,19,21,22,23,24,25,27$,} \\
& $28,29,32,33,36,38,42,44,51,52,56,57,58,64,65$, \\
& $67,68,69,70,78,79,80,83,84,85,86,87,91,92,96$, \\
& $100,102,103,105,106,107,108,109,110,115,116$, \\
& $117,118]$ \\
Categorical task skills & {$[4,7,19,20,23,24,34,37,39,41,43,48,51,52,54$,} \\
& $56,57,63,68,70,71,72,73,74,82,88,90,93,98,99$, \\
& $101,104,110,113,114,120]$ \\
\hline
\end{tabular}

\subsubsection{Skill determinants}

In Table 2, we identify six different skill determinants and classify the corresponding papers according to the respective skill class. The skills of a person can be determined by the age or the experience of the employee, the degree of technical knowledge he acquired or the licenses and qualifications he received. The first three determinants in Table 2 (the age, the experience and the degree of technical knowledge) belong to the hierarchical class. The fourth determinant in Table 2 (licenses, qualifications or job title) belongs to the categorical class. Therefore, a worker with skills determined by the third determinant (Degree of technical knowledge/capability) can perform a certain task better/worse than a worker with a lower/higher degree of technical knowledge. A worker with skills determined by the fourth determinant can do other things compared to a worker with other licenses/qualifications/job title. For determinants two to four, some papers can be found in both the hierarchical and categorical skill class $[51,52,56,57,70,110]$. This is because these authors consider the hierarchical and categorical skill class at the same time and assume that there exists a hierarchical structure in each skill category (see Section 3.1.1). This does however not mean that determinants one to four can belong to both skill classes.

In health care applications, the experience and capability of a nurse is never used directly to formulate a mathematical model of the problem. Instead, the grades of the nurses are used to model their skills. The grade is defined by the experience and education of the nurse and is based on certain national standards [16]. Moreover, each grade determines a specific task set that a nurse of that grade should perform. As these task sets are hierarchically ordered based on the grade, the nurse grading system creates hierarchically ordered categories which is different from all other determinants. Because no research paper defines the grade of a nurse explicitly in the problem description, we added nurse grades as a separate category. As Table 2 shows, the nurse grade is categorized under the hierarchical class. 
Table 2: Different determinants of skills

\begin{tabular}{|c|c|c|}
\hline & Hierarchical class & Categorical class \\
\hline Age/seniority & {$[68,92,110]$} & \\
\hline Experience & $\begin{array}{l}{[16,17,29,36,38,42,51,52,} \\
56,58,64,65,78,85,92,105, \\
106,110,115,116,117,118]\end{array}$ & {$[51,52,56,110]$} \\
\hline $\begin{array}{l}\text { Degree of technical knowl- } \\
\text { edge/capability }\end{array}$ & $\begin{array}{l}{[38,42,44,57,58,78,85,86,} \\
92,102,105,106,107,108,115, \\
116,117,118]\end{array}$ & {$[57]$} \\
\hline $\begin{array}{l}\text { Licenses/qualifications/job } \\
\text { title }\end{array}$ & {$[51,52,56,70,110]$} & $\begin{array}{l}{[4,7,37,41,43,48,51,52,56,} \\
63,70,72,73,82,88,90,93,99, \\
101,104,110,113]\end{array}$ \\
\hline Nurse grade & $\begin{array}{l}{[1,2,3,9,10,11,16,17,22,} \\
25,27,32,33,64,67,69,87,91, \\
103]\end{array}$ & \\
\hline Other & {$[21,22,65,92,109]$} & {$[54,120]$} \\
\hline
\end{tabular}

Note that we make a difference between the level of seniority and experience. While the level of experience depends on the number of times an employee has performed certain tasks, the level of seniority only depends on the time the employee is already present in the company. Majozi and Zhu [92], for example, use the level of seniority in combination with the health of an employee (depending on the number of days he reported himself sick) and his availability (depending on the distance between his home and his office) to calculate the potential performance of a new manager. They use fuzzy set theory to calculate a value which they call the grade of the new manager. Hanne et al. [68] use the age of a driver to determine the minimum rest interval between two rides. They assume that older drivers require longer rest intervals.

Table 2 also shows that other determinants exist such as health, gender, general cognitive ability (GCA) [77] and even the degree of disability of handicapped persons [21]. Some papers do not give a clear definition of the skill determinants at all and present a generic model where skills are user-definable. When the skill class is undefined, such as in $[15,49,55,66,76]$, the skill determinants are also undefined in the respective paper. However, in several cases, only the skill class is defined while the skill determinants are to be defined by the user $[12,19,20,23,24,28,34,71,74,79$, $80,83,84,96,98,100,114]$.

The determinants in Table 2 can have a fixed value for each employee or they can depend on other decision variables in the model. The experience can for example depend on the assignments of tasks to workers. Huang et al. [76] and Bhadury et al. [23] propose a model in which workers have user-definable skills that can change through training. The model, however, lacks the implementation of the consequences of a change in skill level because of the training. In Section 3.3 and Table 7, the different possibilities to change the skills of a person (such as training) are investigated.

Table 3 lists the papers that model the skills as individual and team skills. For 
individual skills, the model comprises the skill level for each single employee. When group skills are considered, the model also (or only) looks at the skill level of a team of workers. When a paper appears in both categories in Table 3, the proposed model also makes decisions about the composition of the teams. In most cases, the sum of all individual skills results in the total pooled skill level of the team. Firat and Hurkens [56], for example, assume that a task can be processed by a group of technicians, provided that the collective capabilities of this group are above a certain threshold. Only a limited number of papers (e.g. Bhadury et al. [23]) model the synergetic effect of pooling differently skilled persons in a team. While Bhadury et al. [23] do incorporate the synergetic effect, they are only concerned with maximizing the diversification in a team and fail to include some skill consequences like task restrictions or performance consequences (see Table 4).

Table 3: Individual or team/group skills

\begin{tabular}{ll}
\hline Individual skills & {$[1,2,3,4,7,9,10,11,12,15,16,17,19,20,21,22,23,24,25$,} \\
& $27,28,29,32,33,34,36,37,38,39,41,42,43,44,48,49,51,52$, \\
& $55,56,57,58,63,64,66,67,68,69,70,71,72,73,74,76,78,79$, \\
& $80,83,84,86,87,88,90,91,92,93,96,98,100,101,102,103$, \\
& $105,106,107,108,109,110,113,114,115,116,117,118,120]$ \\
Team/group skills & {$[23,54,56,63,65,67,69,72,82,99,104,107]$} \\
\hline
\end{tabular}

\subsubsection{Skill consequences}

Table 4 presents the different consequences of skills. We identified five main elements that can be affected by the skills and/or skill level of a person: the labor costs, the speed of work/efficiency, the quality of work, the tasks that he or she can perform and the resulting flexibility.

It may sound obvious that higher skilled or higher educated people induce higher labor costs, but not all papers incorporate this in their model. Row 1 in Table 4 shows the authors who adjust the labor costs based on the skills of the employees. Other researchers even assume that temporary workers have less skills than the permanent workers and therefore entail lower labor costs [38, 44]. Lagodimos and Leopoulos [83] and Lagodimos and Mihiotis [84] also assume that temporary workers have less skills than permanent workers, but they do not take into account a difference in wages.

While higher skilled persons may involve higher labor costs, they also can increase the speed or quality of the produced work. Restrictions concerning the speed or quality of work can be easily formulated as constraints in a mathematical model. In the work of Tiwari et al. [108], for example, a task can only be finished when a certain level of quality is reached. This is only possible when the right worker is assigned to the right task with the appropriate skill level. The speed or quality of work can also be an objective that needs to be optimized in the objective function. Wu and Lee [115] and $\mathrm{Wu}$ et al. [117], for example, try to minimize the total completion time of projects where the speed of work is dependent on the skills of the assigned workers. Eitzen et al. [52] and Fowler et al. [57] assume that, depending on the skill level of a 
worker, a worker is equivalent to one or more workers. Hence, the skill level defines the efficiency of the worker.

Table 4 indicates that many papers incorporate task restrictions resulting from the skills of a person in their model. Certain tasks can require specific skills defined by law or by the company itself. Some papers do not really contain task restrictions since they only want to cover the demand for different skills in each period of the planning horizon. Because people possess different skills, these coverage constraints impose restrictions on the type of demand a person can cover. Therefore, we also list those papers under task restrictions.

Table 4: Consequences of skills

\begin{tabular}{lll}
\hline & Hierarchical class & Categorical class \\
\hline Costs & {$[10,12,22,28,29,38,44,57,58$,} & {$[57,71,88]$} \\
& $91,92,102,106,107]$ & \\
Speed of work/Efficiency & {$[21,24,38,42,44,52,57,58,70$,} & {$[24,43,52,57,70,88,110]$} \\
& $78,85,86,92,105,108,109,110$, & \\
& $115,116,117,118]$ & \\
Quality & {$[15,92,107,108]$} & {$[15]$} \\
Task restrictions & {$[1,2,3,9,10,11,12,16,17,19$,} & {$[4,7,19,20,24,34,37,39,41$,} \\
& $22,24,25,27,29,32,33,36,49$, & $43,48,49,51,52,54,55,56,57$, \\
& $51,52,55,56,57,64,65,66,67$, & $63,66,68,70,71,72,73,74,76$, \\
& $68,69,70,76,79,80,83,84,87$, & $82,88,90,93,98,99,101,104$, \\
& $91,92,96,102,103,106,110]$ & $110,113,114,120]$ \\
& {$[1,2,3,9,10,11,12,15,19,24$,} & {$[4,7,15,19,20,24,34,37,39$,} \\
& $25,27,29,32,33,36,38,42,44$, & $41,43,48,51,52,54,56,57,63$, \\
& $51,52,56,57,58,64,65,67,69$, & $70,71,72,74,76,88,93,101$, \\
& $70,76,78,79,80,85,86,87,91$, & $110,113,114,120]$ \\
& $92,96,102,103,105,106,107$, & \\
& $108,109,110,115,116,117,118]$ & \\
\hline
\end{tabular}

As Table 4 also classifies the papers according to the respective skill class, we see that the costs, the efficiency and quality are the most frequent consequences of hierarchical skills. Since the age, experience and technical knowledge are the three most important hierarchical determinants (see Table 2), the costs, efficiency and quality are usually determined by these three skill determinants. Categorical skills make no difference in performance level and assume that the skills of one category are not better or worse than the skills of another category. Therefore, the speed of work/efficiency, the quality of work and the costs are normally not affected by those skills. However, as Table 4 shows, $\mathrm{Li}$ and $\mathrm{Li}$ [88] and Corominas et al. [43] also consider the impact of categorical skills of the workers on the efficiency. This is because these authors assume that when a worker who normally performs a certain task must suddenly perform another task (for which he is also licensed), he will be less efficient. Hence, when 
workers are required to perform tasks other than their core tasks, their efficiency can decrease while in theory it should be the same. This phenomenon can occur when the workforce is cross-trained and, hence, workers with specific skills are suddenly allowed to perform tasks that are normally assigned to workers with other skills. Besides this negative impact on efficiency, the resulting flexibility is always a positive consequence of cross-training and substitution (see Section 3.2). Table 4 shows the research papers that incorporate flexibility as a consequence of skills. When certain workers are allowed to perform the tasks of other workers, flexibility increases. This allows the company to better respond to an irregular fluctuating demand. These indirect effects of workforce decisions are the subject of the next section.

\subsection{Skill substitution and cross-training}

Substitution of hierarchical skills occurs when workers with a certain skill level are allowed to perform tasks that are normally assigned to workers with a lower skill level. Since categorical skills do not have a hierarchical character, substitution of categorical skills is not possible. Instead, the workforce can be cross-trained such that workers with specific skills are allowed to perform tasks that are normally assigned to workers with other skills. Such workers are often referred to as flexible workers. Substitution and cross-training are easy ways to increase the flexibility of the workforce such that the company can cope with unexpected demand peaks without layoffs or hiring expensive new or temporary workers. Campbell [35] even concludes that flexibility resulting from cross-training can be more valuable than perfect information about the demand, especially when demand variability is high. Despite all advantages, substitution and cross-training requires a certain co-operation and goodwill of the employees which may come at a high cost. For example, when substitution is allowed, higher educated people suddenly have to perform inferior tasks that are normally performed by less educated workers.

In this section, we investigate the different consequences of substitution and crosstraining under different circumstances. Researchers and managers must be aware of the different, possibly negative effects that substitution and cross-training may present. Many papers try to incorporate the dynamics of substitution and crosstraining explicitly. Marentette et al. [93], however, model the effects of cross-training implicitly in their model without deducing the resulting flexibility from calculations or simulations. They assume that the input/output ratio will decrease by $8.5 \%$ (according to the research of Pinker and Shumsky [97]) when the workforce becomes cross-trained. This increase in resource productivity arises because of the added flexibility of the workers. More work can now be done with the same number of workers in the same amount of time. Note the important difference between productivity and efficiency. While flexibility increases the productivity, the efficiency of the flexible worker can decrease. Because workers are now cross-trained, they can perform more tasks and their idle time will be lower. Hence, more output can be obtained with the same number of workers. But as will become clear below, the efficiency of the flexible worker will decrease when he deviates from his core tasks. Hence, the efficiency refers to the performance of the flexible worker compared to the performance of a specialized worker. 
Hierarchical skills and substitution

When skills have a hierarchical nature, higher skilled workers can perform the work of lower skilled workers but not vice versa. When higher skilled workers perform tasks that are usually performed by lower skilled workers, the resulting efficiency tends to be higher. Some papers therefore assume that the speed of work is higher when substitution is allowed $[42,44,58,78,85,86,92,100,105,108,109,115,116,117$, 118]. Tiwari et al. [108] even assume that the quality of work will be higher in this case. Most other papers do not implement an impact on performance because of substitution and are only interested in task restrictions imposed by the required skills. None of the research papers in the first column of Table 5 considers a decrease in performance in case of substitution. Recall that when workers must deviate from their core tasks, the performance can decrease (in case of categorical skills). However, according to the existing literature, it is not believed that substituting hierarchical skills decreases performance because of this reason.

While the substitution of hierarchical skills apparently does not involve the same problems as deviating from core tasks in case of cross-training, it faces another problem with huge consequences. When higher skilled persons must perform tasks designed for lower skilled persons, downgrading occurs. Even without a change in wages this is conceived as being demeaning. Bard and Purnomo [10] incorporate this problem in their mathematical model to solve the preference scheduling problem for nurses with downgrading. Brucker et al. [27], Burke et al. [33] and Pastor and Corominas [96] also incorporate the preferences of the workers to work shifts that require their primary skills. Buchan and Dal Poz [30], Currie et al. [46] and Branson et al. [26] consider the problem of skill dilution and conclude that obtaining a cheaper skill mix with downgrading may be no more cost effective because of the various hidden costs. For example, querulous employees tend to have higher absence and turnover rates [45]. Bard and Wan [12] recognize the problems with downgrading, but do not incorporate them in their model. The model presented by Bard and Wan [12] is special because it uses a substitution matrix that exactly defines the allowed substitution possibilities to avoid such problems. Also Burke et al. [32], Bilgin et al. [25] and Smet et al. [103] assume that nurse grades are not hierarchically overlapping by default. They even allow that a regular nurse can temporarily fill in for a head nurse, as long as no decision making tasks are scheduled.

Operations research papers considering hierarchical skills almost never consider the scenario without substitution (except for $[22,23,83,84]$ ). Lagodimos and Leopoulos [83] and Lagodimos and Mihiotis [84], for example, assume that a certain number of skilled workers should always be present to assure a certain quality and to supervise the unskilled workers. Skilled workers cannot be used as unskilled workers.

Categorical task skills and cross-training

Table 6 gives an overview of the papers that consider cross-training and skill specialization in their model. In an environment with categorical skills, people with different skills can do different tasks, but there is no performance difference between two employees with different skills. When the workforce is cross-trained, people can perform different tasks that differ from their normal (or core) tasks which can lead to a decrease in performance $[47,88]$. Corominas et al. [43] even assume different 
efficiencies for each worker for each different task. Zülch et al. [120] also incorporate the consequences of deviating from the core task of a worker, but the authors do not directly model its impact on the performance. Instead, their model prefers to assign workers to their core task instead of other tasks. Avramidis et al. [7] incorporate the advantages of specialization in their model for staffing and scheduling in call centers. They assume that in practice a given agent often works more efficiently (faster) when handling a smaller number of call types (i.e., if his/her skill set is artificially reduced). Ertogral and Bamuqabel [54], however, do not incorporate any disadvantages of flexible cross-trained workers in their model to solve a similar workforce planning problem in a call center.

Marentette et al. [93] draw attention to the fact that a model should consider both the downsides of cross-training and the advantages. Above all, these downsides must include the high training costs incurred in order to qualify workers to perform multiple tasks. Pinker and Shumsky [97] assume that while specialized workers can perform higher quality work because of a greater level and complexity of experience, crosstrained workers save money through lower staffing levels. Also Chakravarthy and Agnihothri [40] report that a system of $100 \%$ cross-trained workers is only beneficial when the disadvantages are limited. Maintaining a mix of flexible and specialized workers is best for balancing the advantages and disadvantages [95].

While operations research papers considering hierarchical skills almost never consider the scenario without substitution, it is more common to assume specialization of the workforce for papers considering categorical skills (see Table 6). In such scenarios, it is often the case that the deployment of flexible cross-trained workers is impossible from a practical point of view. Song and Huang [104], for example, work with department specific skills. Since the different departments are located too far from each other, it is impossible to make the workers change department to perform another task.

Table 5: Hierarchical skills and substitution

\begin{tabular}{ll}
\hline Substitution & {$[1,2,3,9,10,11,12,19,21,24,25,27,29,32,33,36,38,42,44,51$,} \\
& $52,56,57,58,64,65,67,69,70,78,79,80,85,86,87,91,92,96,102$, \\
& $103,105,106,107,108,109,110,115,116,117,118]$ \\
No substitution & {$[22,23,25,28,32,83,84,103]$} \\
\hline
\end{tabular}

Table 6: Categorical skills and cross-training

\begin{tabular}{ll}
\hline Cross-training & {$[4,7,19,20,34,37,39,41,43,48,51,54,57,63,70,71,72,74,88$,} \\
& $93,101,110,113,114,120]$ \\
Skill specialization & {$[23,24,52,56,73,82,90,98,99,104]$} \\
\hline
\end{tabular}




\subsection{Adapting the skill pool}

While there are some differences between hierarchical and categorical skills in how they affect the performance of the workforce when substitution or cross-training is introduced in the model, there is no difference between the two categories regarding the different ways to adapt the skill pool. In this section we investigate the different possibilities used in the literature to adapt the composition of the skill pool in workforce planning problems. An overview of the respective papers is given in Table 7. Again, we focus on the impact that certain skill decisions have on the workforce planning problem, i.e., the impact on the costs, speed and quality of work, task restrictions and flexibility.

Table 7: Adapting the skill pool

\begin{tabular}{ll}
\hline Hire/dismiss temporal workers & {$[11,24,28,38,42,58,69,70,80,83,84,90,91,105,106]$} \\
Hire/dismiss new/old workers & {$[10,15,29,38,44,57,58,63,76,88,91,98,104,120]$} \\
Training & {$[16,17,37,57,76,88,93,104,108,113,120]$} \\
Learning/forgetting & {$[24,38,44,76,78,85,100,105,115,116,117,118]$} \\
Voluntary leaving & {$[76,104]$} \\
Hire volunteers & {$[65,101]$} \\
\hline
\end{tabular}

Hire/dismiss temporal workers

Hiring and dismissing temporal workers is an easy and popular way to increase flexibility. The research papers shown in the first row of Table 7 assume that temporal workers are inferior to permanent workers in several ways. Some of them assume that temporal workers have less skills and therefore entail lower labor costs than permanent workers $[28,38,58,106]$. Others mention the lower efficiency or the slower learning rate of temporal workers as their main disadvantages [24, 38, 42, 58, 105]. Some authors assume no difference in performance between temporal and permanent workers and are only concerned with task restrictions in their model [11, 83, 84]. According to Heimerl and Kolisch [70], the temporal workers can be more or less efficient than the internal workers. An interesting fact is that these authors use a constraint in their model to ensure a minimum ratio of the work performed by internal resources to the work performed by temporal (external) resources. This way, core competencies can be kept within the company, the management of the project can be performed internally, and a minimum of internal knowledge will be employed. In general, all researchers allow for the increase in flexibility as a result of working with temporal workers. Corominas et al. [42], for example, use this flexibility to cope with a highly seasonal demand.

Hire/dismiss new/old workers

In contrast to newly hired temporal workers, no difference in performance is reported in the literature between newly hired permanent workers and the current workforce. Therefore, all related papers assign newly hired workers the same labor 
costs in their model. Hiring and dismissing permanent workers is the most expensive option, but of course allows to tune the workforce to the demand. Despite the high costs, this can sometimes be a viable option when the disadvantages of substitution are considered. Recall from Section 3.2 that flexibility can also be obtained by substitution and cross-training. $\mathrm{Li}$ and $\mathrm{Li}$ [88], for example, explicitly model the cross-training cost of a worker to create flexibility and the cost of hiring a new permanent worker. Furthermore, they incorporate the possible decrease in efficiency caused by letting the workers deviate from their core task (see Section 3.2) to compensate for the high cost of hiring a new permanent worker. This way, a trade-off is made by the model between flexibility, efficiency and costs to choose between hiring a new permanent worker or cross-training the workforce.

Training

Training is a straightforward way to enhance the skills of a person in order to increase his performance (quality, speed of work and efficiency) or to allow the worker to perform a different task. The latter is referred to as cross-training and is used when categorical skills are considered. A good model must always contain the training cost (loss of available working time and/or monetary costs) and the possible benefits (the increased flexibility or performance). While learning happens automatically, training must be planned. Hence, decisions must be made by the workforce planning model to determine who should receive training and when this should take place.

In $[57,76,88,93,104]$, the planning decision for cross-training the workforce is incorporated in the model. A decision is made about how many employees should be trained to acquire certain skills. Li and Li [88], Song and Huang [104] and Fowler et al. [57] make these decisions on a weekly or monthly basis, but Li and $\mathrm{Li}[88]$ is the only paper that does take the disadvantages of cross-training into account. Huang et al. [76] and Marentette et al. [93] only make the training decision on a yearly basis and therefore fail to provide the exact timing of the training. Tiwari et al. [108] and Zülch et al. [120] neglect the training decision during the workforce planning optimization problem and only afterwards analyze the bottleneck skills. In a second phase, they provide an advice to the management about what skills should be trained. According to Hopp and Oyen [75], cross-training can also lead to a synergetic effect: employees get a holistic view of the company which aids the internal communication as well as the invention of new innovative ideas and increases the learning effect and the loyalty of the employees. These positive indirect effects can help mitigate the negative effects of cross-training. Including the precise timing and consequences (positive and negative) of training tends to be a difficult task when one looks at the relevant literature.

Training can take place on the job or externally. In the technical operations research literature, the type of training (on the job or external) is always fixed instead of a decision variable. Training on the job is preferred in most cases because of the direct link with the company and the lower costs. However, according to du Boulay and Medway [50], training on the job is very difficult these days because of several reasons. First, for some tasks, it is prohibited by law to involve people without the necessary skills or qualifications. Therefore, Beddoe et al. [16] and Beddoe and Petrovic [17] talk about eye-training instead of on the job training. Some researchers incorporate the training decision in the model, but fail to consider the effects of the 
training on the skills of the employees $[16,17,37]$. Second, because of high work pressure, it might be impossible to involve people that slow down the work process. And third, according to Markes [94], theoretical (academic) knowledge nowadays becomes more and more important compared to practical knowledge.

Finally, we found that the skill related workforce planning literature lacks some important features concerning training. First, the most direct effect of training, i.e., the reduced availability or even total absence of the worker during his training period is never incorporated in the model. As such, the trade-off between absence due to training and creating more flexibility has never been explicitly studied in the literature. Second, when researchers dedicate special attention to the planning of training, no attention is paid to the negative effects of cross-training.

Learning/forgetting

While training must be planned, learning happens automatically. Argote [6] and Yelle [119] specify learning as learning by doing. Forgetting occurs when a worker does not perform certain tasks for a certain period of time. Row 4 in Table 7 shows the different papers that incorporate learning in their model to increase the performance of the workforce. Most papers only consider a constant learning rate and increase the skill level as time passes or tasks are repeatedly performed. Wu and Lee [115] and Wu et al. [117] also assume that the learning rate depends on the current skill level of an employee. On top of the learning effects, Xingong and Guangle [118], Lee and $\mathrm{Wu}$ [85] and Sayin and Karabati [100] also consider the effects of forgetting and call this the deteriorating effect. To overcome the problems of forgetting, they make use of group theory. Their model tries to group similar tasks as much as possible in order to increase the learning effects and decrease the effects of forgetting.

While many papers take into account learning or forgetting effects, it is striking that most researchers do not devote sufficient attention to the realism of the methodology to model learning and forgetting. In many cases they assume for example that the learning rate is linear. Only a limited number of researchers use more complex and more realistic learning curves [24, 38, 100, 105].

\section{Voluntary leaving}

Row 5 in Table 7 shows the research papers that account for employees that leave voluntarily, also called turnover. Employees tend to leave when they get dissatisfied as a result of bad decisions. Huang et al. [76] and Song and Huang [104] do not link the voluntary leaving of people to decisions in the model, but just assume a fixed percentage of people that will leave voluntarily. Hence, an interesting path for future research is to incorporate voluntary leaving as a negative consequence of skill substitution.

\section{Hire volunteers}

Scott and Sampson [101] consider the use of volunteers to increase the flexibility of the company and give some insights into the dynamics of working with volunteers. Two important issues are raised to take into account when incorporating volunteers in the problem. First, volunteers that are hired must be utilized as much as possible because utilized labor is more likely to volunteer again in the future compared 
to unutilized labor. While the utilization of hired volunteers is important, overutilization will decrease the availability of future volunteers. Second, the amount of work that they are willing to perform (i.e., the committed labor) is directly linked to the perceived quality of the tasks assigned to the volunteer. Gordon and Erkut [65] only consider volunteers for the scheduling of a yearly recurring event. They stress the importance of the preferences of the volunteers to make them come back every year. Therefore, their objective is to maximize volunteer preferences subject to several coverage constraints.

\section{Technical aspects of skill related workforce planning}

Until now, we mainly dealt with the managerial aspects of workforce planning incorporating skills. In this section, we explore the technical implications of incorporating skills in workforce planning problems. First, we investigate the impact of incorporating skills in a workforce scheduling problem. Next, we focus on the different modeling techniques that are used in the literature to compose mathematical models to represent the workforce problem with skills. Finally, we look at the solution techniques proposed by different researchers to solve the problem.

\subsection{Impact of incorporating skills}

Workforce planning problems are, in general, very difficult to solve, even when they are tackled in a simplified version containing only a single criterion and homogeneous skills [34]. Moreover, the problem is known to be NP-complete [14]. When skills are incorporated into a workforce scheduling problem, the workforce becomes heterogeneous instead of homogeneous. Differences emerge between individual workers. This means that only certain workers are capable or allowed to do certain things. Moreover, assigning certain workers to certain tasks can have a different impact on the objective value. The impact of incorporating skills on the complexity of the problem depends on the type of decision that is made by the model. A different impact can be observed between models that decide on the number of workers that must be present during certain time slots or that must be assigned to certain tasks and the models that decide on the timing or assignment of each individual worker.

The first type of models are only concerned with the number of workers that must be present or assigned while the second type of models make decisions for each single worker. By incorporating skills in the first type of models, the number of decision variables increases. One now has to decide on the number of workers of each type (with certain skills) that must be available or assigned. Since the incorporation of skills often entail extra coverage constraints (for each skill) and/or task restrictions, the number of constraints will also increase. An increase in the number of decision variables usually leads to an increased complexity and required computation time. Ertogral and Bamuqabel [54] demonstrate this by comparing a staff scheduling problem with and without skills.

When skills are incorporated into a model that decides on the timing or assignment of each individual worker, the impact is different. Of course, the variant with a homogeneous workforce of this second type of models already have more decision variables than the first type of problems since there is a timing/assignment variable for 
each worker. Incorporating skills in this case also increases the number of constraints, but does not increase the number of decision variables. However, the incorporation of skills has an additional impact on the complexity of this type of problems compared to the first type of problems. Since each individual worker is different in the heterogeneous case, assigning different workers to specific time slots or tasks can have a different impact on the objective value or can even be forbidden by certain constraints. This implies that symmetry disappears which increases computation time. As a positive consequence, adding more skills will not increase the number of decision variables in this type of models and does not affect computation time drastically. The number of workers on the other hand will have a significant impact on complexity $[52]$.

Symmetry exists in a linear program if its variables can be permuted without changing the structure of the problem [59]. In the case of workforce planning, symmetry arises when the model contains different variables for each worker, while those workers are in fact similar to each other. In that case, there is no difference in assigning worker 1 or worker 2 to a certain job. The problems with symmetry come from the fact that many sub-problems in the enumeration tree are equal, leading to a wasteful duplication of effort [59]. A recent survey of techniques developed in the constraint programming community to solve symmetric problems is provided by Gent et al. [60]. Symmetry should not be a problem when handled correctly. It has even been exploited in mathematical programming in order to speed up solution methods [89]. The first step is always to detect symmetries and the second step is to compose a solution technique to avoid symmetry problems. An example of a research paper that successfully removes symmetry in order to solve the airline crew scheduling problem is Vance et al. [112]. When skills are introduced into the problem and the workforce becomes more heterogeneous, symmetry disappears. This eliminates of course the need for difficult techniques to remove symmetry, but unfortunately also eliminates the possible speed up in computation time.

\subsection{Skill modeling techniques}

Incorporating skills entails that the assignment of different workers to specific time slots or tasks can have a different impact on the objective value or can even be forbidden by certain task restrictions. This has of course implications for the objective function and constraints in the model. The goal of the next two subsections is not to analyze the impact of skills on the complexity of the model such as in Section 4.1, but rather to demonstrate how the skill dynamics (as outlined in Section 3) can (or should) be modeled in order to construct a realistic model. The analysis also shows how the negative effects of some managerial decisions (such as substitution) can be incorporated and mitigated in the model.

From Table 4 in Section 3.1.3 we can deduce that there are two possibilities to incorporate the consequences of skills in a mathematical model to represent a workforce planning problem. First, skill related decisions can have an effect on the performance and efficiency of the workers. Recall that the speed and quality of work and the costs are three consequences that are frequently used in operations research papers. Second, certain tasks can require a certain skill level which adds specific skill related constraints to the model. These two ways of incorporating the skill consequences in 
a mathematical model can each be modeled in two different ways. The first way is to optimize the workforce planning problem under specific skill related constraints. The second way is to put skill based measures in the objective function of a mathematical problem. Table 8 gives an overview of how the relevant research papers can be classified in this way.

Table 8: Skill modeling

\begin{tabular}{lll}
\hline & Skills in constraints & Skills in objective function \\
\hline Task restrictions & {$[1,2,3,4,7,9,10,11,12,15,16$,} & {$[32,51,66,67,68,71,80,88,90$,} \\
& $17,19,20,22,24,25,27,29,32,33$, & $91,92,98]$ \\
& $34,36,37,39,41,43,48,49,51,52$, & \\
& $54,55,56,57,63,64,65,68,69,70$, & \\
& $72,73,74,76,79,82,83,84,87,93$, & \\
& $96,99,100,101,102,103,104,106$, & \\
& $108,110,113,114,120]$ & {$[21,28,38,44,58,78,85,86,88,92$,} \\
Skill based perfor- & {$[24,42,43,52,57,70,107,108]$} & $100,105,106,108,109,110,115$, \\
mance/efficiency & & $116,117,118]$ \\
measures & & \\
\hline
\end{tabular}

\subsubsection{Skills in Constraints}

One should first decide to model the skills in the objective function or the constraints. Of course, task restrictions are more often modeled in the constraints while skill based performance/efficiency measures are often modeled in the objective function (see Table 8). Since skill based performance/efficiency measures usually have a direct effect on the objective value (such as is the case for speed and quality of work) they are modeled in the objective function most of the time. However, some researchers like Tiwari et al. [108] model a skill based performance measure in the constraints instead of in the objective function. In this particular paper, there is a hard constraint on the final quality of a job. Only when a certain level of quality is reached, the job can be finished. This level of quality can only be obtained by assigning certain workers during a certain amount of time to the task. Of course, the time that is needed to obtain the required quality depends on the skill level of the worker. In Corominas et al. [42], there is a hard constraint on the maximum time that is needed to finish a task while the speed of work of each worker depends on his skill level. Corominas et al. [43] puts the efficiency (a value between 0 and 1 multiplied by the availability of the worker) of each worker of each skill category for each task type in the constraints to model the task coverage constraints. Furthermore, the authors assume that not all tasks can be performed by every skill category. Therefore, Corominas et al. [43] appear twice in the first column in Table 8. For the same reason, Eitzen et al. [52], Fowler et al. [57] and Heimerl and Kolisch [70] also appear two times in the first column because they assume that workers with a higher skill level are more efficient. Hence, the efficiency of the worker is integrated into the 
coverage constraint.

To model task restrictions in the constraints of a mathematical model, researchers use a variant of model (1) - (2) [82]. In this model, constraint set (2) ensures that only workers with the required skills are assigned to a certain task. Of course, the set of workers $W$ and the set of persons $P_{t}$ that can perform task $t$ can be variables in the model depending on decisions regarding staffing, training, learning and substitution. Furthermore, extra constraints can be added to make sure that each worker is only assigned to one task.

Generic model to incorporate task restrictions

$$
\begin{aligned}
& t \in T: \quad \text { set of tasks that need to be performed } \\
& w \in W: \quad \text { set of workers that are available to perform tasks } \\
& P_{t}: \quad \text { set of persons that can perform task } t \in T\left(P_{t} \subseteq W\right) \\
& x_{t w}= \begin{cases}1 & \text { if task } t \in T \text { is assigned to worker } w \in W \\
0 & \text { otherwise }\end{cases}
\end{aligned}
$$

Optimize:

Subject to:

$$
\text { Objective function }
$$

$$
\sum_{w \in P_{t}} x_{t w}=1, \quad \forall t \in T
$$

\subsubsection{Skills in Objective function}

When skills are modeled in the constraints such as in the previous section, we speak of hard constraints. Hard constraints can never be violated in a feasible solution. The alternative is to model the constraints in the objective function by penalizing constraint violations. By using such a penalty function, constraint violations are penalized but cannot always be avoided. In this case, we speak of soft constraints. Several researchers use these soft constraints instead of hard constraints to facilitate the exploration of the search space in a local search heuristic. When skills are incorporated, many task (and other) restrictions are added to the problem which makes the search quite restrictive. Hanne et al. [68] demonstrate this by comparing a model with task restrictions in the constraints and a model with task restrictions in the objective function. Ho and Leung [72] also recognize this issue and propose complex tunneling procedures to deal with the many skill constraints.

In general, using constraint violation penalty functions instead of hard constraints in the model leads to a possible less precise and inferior result. Concerning skill dependent task restrictions, the use of such a penalty function instead of a hard constraint can, however, result in a far more realistic model that captures more of the skill dynamics (as discussed in Section 3). This way, the negative effects of some managerial decisions (such as substitution) can be incorporated and mitigated in the model. Majozi and Zhu [92], Günther and Nissen [66], Sadjadi et al. [98], Hanne et al. [68], Eiselt and Marianov [51], Knust and Schumacher [80] and Gutjahr and 
Rauner [67], for example, try to minimize the mismatch between the skills of the person assigned to a certain task and the required skills to perform this task. This way, Eiselt and Marianov [51] try to avoid boredom. Because Majozi and Zhu [92] also incorporate the costs associated with different skill levels, their model will make a trade-off between the higher costs of the skill level of a worker and the performance of the worker based on his skill level. Hence, the model will not choose for the cheapest option because this will decrease the performance of the company, but it will also not choose for the most expensive option because the costs cannot outweigh the increase in performance. $\mathrm{Li}$ and $\mathrm{Li}[88]$ do something similar and minimize the extent of deviations from the workers core tasks in the objective function. This way, a trade-off can be made between the positive effect of the cross-training (the effects of an increase in flexibility of the workforce) and its negative effects (see Section 3.2). Hence, the model will prefer the assignments of tasks to employees that do not differ from their core task. Of course, some task restrictions can never be violated (concerning safety issues for example) leading to a combination of hard and soft constraints such as in the model of Burke et al. [32].

In $[28,38,44,58,78,85,86,105,109,115,116,117,118]$, the authors model the skills in the objective function, but not as soft constraints. Instead of skill constraints, these papers consider skill based performance/efficiency measures with a direct impact on the objective (such as speed of work and the quality of the finished products).

Model (3)-(4), presented hereafter, shows the generic way of modeling skills in the objective function [108]. While constraint set (2) restricted the tasks that can be assigned to the workers, constraint set (4) entails no such restrictions and only ensures that all tasks will be performed. Each possible assignment of workers to tasks is associated with a certain effect represented by the parameter $\Pi_{t w}$. This is the resulting effect, for instance with respect to quality, from assigning worker $w$ to task $t$ on the objective value. The quality can be of direct interest to the company or can have an indirect effect. Stratman et al. [105], for example, take into account the costs of scrapped products as a result of a bad alignment of skills and tasks (i.e., bad quality). Other definitions of $\Pi_{t w}$ are the time required for worker $w$ to perform task $t$ or the quantity of resources that worker $w$ requires to perform task $t$. The objective function $h()$ in expression (3) is therefore dependent on these effects $\left(\Pi_{t w}\right)$.

Generic model to incorporate skill based performance/efficiency measures

$t \in T: \quad$ set of tasks that need to be performed

$w \in W: \quad$ set of workers that are available to perform tasks

$\Pi_{t w}$ : $\quad$ resulting performance effect from assigning worker $w \in W$ to task $t \in T$

$x_{t w}= \begin{cases}1 & \text { if task } t \in T \text { is assigned to worker } w \in W \\ 0 & \text { otherwise }\end{cases}$ 
Optimization model:

Optimize:

Subject to:

$$
\begin{aligned}
& \text { Objective function } h\left(\Pi_{t w} x_{t w}\right) \\
& \qquad \sum_{w \in W} x_{t w}=1, \quad \forall t \in T
\end{aligned}
$$

\subsection{Solving skill related workforce planning problems}

In this section, we look at the technical procedures proposed by the operations research literature to solve workforce planning problems incorporating skills. First, we analyze how stochasticity is sometimes introduced in the problem. Next, we discuss the different solution techniques to solve the mathematical model.

\subsubsection{Stochasticity in workforce problems}

Real life workforce problems always contain some uncertainty. The demand for products or services is hardly ever known with complete certainty and unexpected changes are always possible. Table 9 summarizes the conducted research that takes into account uncertainty. This table can be used in combination with Table 11 to analyze which solution methods are used to solve which kind of problems. As Table 9 shows, it is very alarming that the number of papers that incorporate uncertainty in their model remains rather limited compared to the papers that investigate deterministic problems. While uncertainty is ubiquitous, it remains a very popular topic for the future research section of many papers.

Table 9: Type of problem: deterministic or stochastic

\begin{tabular}{ll}
\hline Deterministic problem & {$[1,2,3,4,9,10,11,12,15,16,17,19,20,21,22,23,24,25$,} \\
& $27,28,29,32,33,34,36,37,38,39,41,42,43,44,48,49,51$, \\
& $52,55,56,57,63,64,65,66,67,68,70,72,73,74,78,79,80$, \\
& $82,83,84,87,88,90,91,92,93,96,100,101,102,103,106$, \\
Stochastic problem & $108,109,110,114,115,116,117,118]$ \\
\hline
\end{tabular}

Most papers that consider a stochastic problem account for the uncertainty in demand $[7,54,58,69,71,76,86,88,98,99,104,107,113]$. Furthermore, the required workload and required processing time for tasks can be stochastic as elaborated by Stratman et al. [105] and Huang et al. [76]. Stratman et al. [105] also assume uncertainty in scrap costs, labor costs, training costs and, like Song and Huang [104], also allow for uncertainty related to voluntary turnover. As a source of uncertainty, Zülch et al. [120] and Fragnière et al. [58] consider personnel absenteeism which may lead to unexpected shortages of qualified and non-qualified employees. It is clear that only very few authors incorporate uncertainty related to the (effects of the) skills of a worker which is an interesting path for future research. 


\subsubsection{Solution techniques}

Workforce problems of realistic size almost always require special solution techniques to solve the problem in reasonable time. We found only one research paper that can use total enumeration to obtain the optimal solution to the problem [93]. Table 11 presents the different solution techniques that are used in the scientific literature to solve workforce planning problems incorporating skills. Table 10 can be used to distinguish between exact and heuristic methods. (Meta-)heuristic algorithms such as Tabu Search, Genetic Algorithms and Simulated Annealing have been used in some cases to solve real-life employee scheduling problems that would require too much computation time to solve with exact methods [53]. These techniques make it possible to overcome the large computation times of exact models and can deliver comparable results. It has even been proven that heuristic techniques often outperform certain exact techniques for workforce scheduling problems of realistic dimensions [18, 31]. Glover and McMillan [62], for example, used Tabu Search to solve the general employee scheduling problem. Even in solution methods that can be used to provide the optimal solution to the problem, heuristic procedures are sometimes used. Bard and Purnomo [10], for example, use a heuristic approach to solve the pricing problem during column generation because their pricing problem lacks a special structure. Therefore, the traditional exact approach cannot solve the problem in an efficient way. Another example can be found in the work of Sayin and Karabati [100]. They approximate their quadratic objective function by different linear pieces and then use mixed integer programming (MIP) to solve the resulting problem. Hence, the authors obtain a heuristic solution even though the MIP model is solved to optimality. Bhatnagar et al. [24] use linear programming to obtain the optimal (fractional) solution to the problem and apply a rounding algorithm to produce an integer result. Some authors go even further and assume that the management is not interested at all in some (near) optimal solution and only search for a feasible solution to the problem $[16,17,41,79]$. When a heuristic technique is proposed to overcome the large computation time, the advantages of the speed-up should be compared with the loss in solution quality. Wu et al. [117] and Wu et al. [116] therefore compare the results obtained with their exact branch and bound technique to the results obtained with a heuristic algorithm.

Table 10: Exact or heuristic

\begin{tabular}{ll}
\hline Exact & {$[4,12,19,20,23,28,37,42,43,44,51,52,54,55,58,63,65,69,70$,} \\
& $71,76,78,79,84,85,86,88,91,92,93,96,99,101,102,106,108$, \\
& $116,117,118]$ \\
& {$[1,2,3,7,9,10,11,15,16,17,21,22,24,25,27,29,32,33,34,36$,} \\
& $38,39,41,48,49,52,55,57,64,66,68,72,73,74,80,82,83,87,90$, \\
& $98,100,103,104,107,109,110,113,114,116,117,120]$ \\
\hline
\end{tabular}


Table 11: Solution techniques

\begin{tabular}{ll}
\hline Mathematical programming & {$[88,101]$} \\
Goal programming & {$[12,19,20,37,42,54,65,84,91,96,102,108]$} \\
Integer linear programming & {$[24,43,76]$} \\
Non-integer linear programming & {$[4,7,44,49,51,56,58,65,70,74,80,92,99$,} \\
Mixed integer linear programming & $100,106,116,117]$ \\
& {$[10,11,15,28,29,52,91]$} \\
Column generation & {$[82]$} \\
Lagrange relaxation & {$[48]$} \\
Benders decomposition & \\
Heuristics & {$[9,72,103,107,116]$} \\
Simulated annealing & {$[22,25,32,49,72]$} \\
Tabu search & {$[1,2,3,9,34,57,98,110]$} \\
Genetic algorithm & {$[27,39,49]$} \\
Greedy algorithm & {$[7,15,16,17,21,24,29,33,36,38,41,52,55$,} \\
Other & $56,64,66,67,68,73,74,80,83,87,90,103$, \\
& $109,114,120]$ \\
Discrete-event simulation & {$[7,76,88,105,113,120]$} \\
Simulation Optimization & {$[71]$} \\
Constraint programming & {$[16,17,41,79]$} \\
Queuing & {$[54,86]$} \\
Other algorithms & {$[12,20,23,55,63,69,78,85,104,113,118]$} \\
\hline
\end{tabular}

In case the skill determinants directly determine the different tasks a worker can perform (e.g., the degree of technical knowledge or the acquired licenses), the skill consequences are very clear and easy to implement in a mathematical model. When this is not the case, the consequences of the differences in skill level (e.g., the quality or speed of work) must be estimated through empirical observations and interviews. Only a few researchers pay enough attention to the techniques to approximate realistic skill consequences. Majozi and Zhu [92], for example, use fuzzy set theory within the context of integrated planning and scheduling to approximate the potential performance of a worker. Fuzzy set theory is a technique that provides a framework for modeling uncertain or ambiguous information (such as experience, expertise, responsibility and age) that is commonly encountered in industry. The authors also argue that traditional mathematical procedures consequently fail to address this issue effectively. The authors then incorporate the output from the fuzzy set procedure in a mixed integer linear programming formulation to determine the optimal solution to the problem.

According to Table 11, mixed integer programming appears to be the most popular mathematical programming technique to solve this kind of workforce planning problems. While integer linear programming is only concerned with integers and non-integer linear programming is only concerned with non-integer variables, mixed 
integer linear programming considers both at the same time. Completely non-integer programs (such as in $[24,43,76]$ ) are easier to solve, but often fail to give a realistic representation of the real situation.

When heuristics are used, many researchers prefer to provide their own heuristic based on the respective problem structure to quickly find a good solution. Besides the three main meta-heuristics mentioned in Table 11, other heuristics are proposed in the literature such as hill climbing [109], particle swarm optimization [38, 66], scatter search [21, 33, 90] and Ant Colony Optimization [67].

Simulation is mostly used to evaluate the obtained (near) optimal solution in a stochastic environment [76, 88, 120]. In Stratman et al. [105], simulation is only used to compare different decisions without solving a mathematical model. We refer to Section 4.3.1 for information about the stochastic elements in the respective simulation models. In Zülch et al. [120], the results obtained from the simulation experiment are used in a next step to adjust the decisions made in the first step. This process is even repeated for several iterations. The task of simulation is, in this case, to create a decision basis for the best disturbance compensation strategy. To obtain even better results, the simulation information can be directly incorporated in the optimization model in the first step. When the information from simulation is directly used in the main optimization model, we refer to this approach as model enhancement. The term "model enhancement (ME)" was used by Bachelet and Yon [8] to indicate a different way of combining simulation and optimization. While most optimization-simulation couplings focus on improving the objective function evaluated from simulation (like the simulation optimization approach), ME still focuses on optimizing the theoretical objective function. It tries to improve the solution provided by a mathematical model by the use of simulation. Avramidis et al. [7] use a technique that combines simulation, integer or linear programming and cut generation. The authors show that the standard approaches sometimes yield solutions that are highly suboptimal and inferior to those obtained by their proposed method.

The last row of Table 11 shows the research papers that use solution techniques different from all mainstream approaches. Xingong and Guangle [118], Janiak and Rudek [78] and Lee and $\mathrm{Wu}$ [85], for example, compose their own optimization algorithm based on the specific characteristics of their considered problem and prove the optimality of the proposed algorithm. Song and Huang [104] model their problem as a multistage stochastic program and propose a successive convex approximation method which solves the problem in stages. In order to maximize the synergetic effects of heterogeneous teams, Bhadury et al. [23] model the problem as the so called dining problem. In the dining problem, the invited families wish to develop a seating arrangement where it is ensured that at each table there is representation from as many different families as possible. Hence, the social interaction must be maximized. To solve this problem, Bhadury et al. [23] transform this problem into a max flow network problem and apply an exact efficient algorithm. 


\section{Application areas}

In this section, we investigate the different application areas of the published workforce planning research incorporating skills. Table 12 shows a classification of the main application areas we discovered: services, manufacturing, military and general. The category "General" contains all research papers that do not target a specific application area. We make a distinction between services and manufacturing because unlike manufacturing, where standard shifts and days off are the rule, the service industry often operates 24 hours a day, 7 days a week and faces widely fluctuating demand [13]. We also divide the services in more specific application areas because the service area is often complicated by labor laws, union contracts and aperiodic fluctuations in demand that may vary wildly depending on the application area.

In Table 13, we categorize the related research papers on whether they present a real case study in their paper or limit themselves to test sets or no test case at all. A clear distinction is made between the research that makes use of computational tests based on real data and research that uses computational tests based on purely theoretical data generated by the authors. Test sets that are generated using statistical distributions based on real-life data (e.g., averages based on observations) are also considered to be test sets based on real data. As Table 13 shows, the research that is actually applied in practice (i.e., the real case studies) remains rather limited. Fortunately, the number of research papers that do not provide a test of the proposed model also remains limited to a few cases.

Table 12: Application areas

\begin{tabular}{ll}
\hline Services & {$[43,51,63,65,66,74,76,86,101,104,110]$} \\
General & {$[1,2,3,9,10,11,16,17,22,25,27,28,29,32,33,39,41$,} \\
Health care & $55,64,67,69,87,88,91,103]$ \\
& {$[38,56,70]$} \\
Maintenance & {$[7,15,54,56,71,73,107,108,109,113]$} \\
Call center/telecommunication & {$[12,34,37,68,72,79,80,90,93]$} \\
Transportation & {$[21,24,42,44,52,57,78,82,83,84,85,92,105,106,115$,} \\
Manufacturing/production & $116,117,118,120]$ \\
& {$[93,99]$} \\
Military & {$[19,20,23,36,48,49,58,96,98,100,102,114]$} \\
General &
\end{tabular}


Table 13: Real test case or theoretical tests

\begin{tabular}{ll}
\hline Real case study & {$[10,21,22,23,24,25,32,39,49,55,65,69$,} \\
& $79,80,87,90,91,103,105,114,120]$ \\
Computational tests based on real data & {$[1,2,3,7,11,12,16,17,27,28,29,33,34,37$,} \\
& $38,41,42,51,52,63,64,66,67,70,72,73,74$, \\
& $76,83,88,93,98,99,101,109]$ \\
Computational test based on generated data $\quad[9,15,19,20,28,36,41,43,44,48,49,54,55$, & $57,58,68,71,82,84,86,92,96,100,102,104$, \\
& $106,107,108,110,113,116,117]$ \\
No test case & {$[78,85,115,118]$} \\
\hline
\end{tabular}

It is remarkable that many researchers claim that they present a generic model that can be applied to many different settings, while each researcher comes up with a completely different approach. We found that $[15,42,44,56,57,72,78,85,92,93$, $100,109,115,116,117,118]$ present a more or less generic approach to incorporate skills in workforce planning problems. Note that these research papers mainly focus on manufacturing applications. This is not very surprising because this application area is mostly concerned with assigning workers to tasks subject to task or performance restrictions. Therefore, manufacturing is also the most important application area for this kind of research.

As Table 12 shows, Wongwai and Malaikrisanachalee [114] and Bhadury et al. [23] present a general model that is not limited to a specific application area. Their model is however only suitable for areas working in a project structured environment. Huang et al. [76], Firat and Hurkens [56], Drezet and Billaut [49], Heimerl and Kolisch [70], Bellenguez-Morineau and Néron [20] and Bellenguez and Néron [19] also present a model for project based scheduling. While most researchers claim that they present a generic model, some researchers are very specific regarding the domains to which their application can be extended. Lee [86] for example present a general model for a service environment. According to the authors, the model can be applied to the rotating work schedule for parking lot attendants at lots which have a single entryexit point, the exchange department of a supermarket where a group of workers rotate between customer service (e.g., goods exchange, refund) and providing price checks for the different check-out counters and the rotating work schedule for sales personnel at the computer division of a consumer electronic store. Another example is the work of Batta et al. [15]. While this work is focused on call centers, it can also be applied to other examples of service centers such as hospitals (assigning nurses to different wards) and police departments (assigning police officers to different tasks). 


\section{Summary}

In this research, we provide an overview and classification of the relevant operations research literature in order to guide the researcher in his search to find useful papers and information regarding skill related workforce planning problems. The unique feature of this approach is that we not only consider the relevant technical literature, but also the relevant managerial literature.

As it is important for an operations researcher to identify all potential factors that may impact the workforce decisions, we first focus on the managerial aspects of incorporating skills in workforce planning problems. Therefore, we give an extensive definition of skills in the first section of this paper. We identify different classes, determinants and consequences of skills and elaborate the different consequences of substitution and cross-training. Thereafter, the different ways to adapt the skill pool are considered. The final objective of the managerial insights we provide in this paper is to make operations researchers aware of the complex dynamics of incorporating skills in workforce planning problems and to promote the development of more realistic and applicable mathematical models.

In the next sections, we investigate the technical details of skill related workforce planning as we try to point the interested researcher to useful information related to his research. We first look at the different ways to model skills in mathematical problems (in the constraints and objective function) and formulate the two respective generic mathematical models. Next, our focus is on the variety of solution techniques proposed in the literature where we make a difference between heuristic and exact solution approaches.

Finally, the different application areas are explored and we discus the possible ways that researchers use to validate or test the proposed models.

\section{Conclusion and discussion}

Based on the evidence found in the reviewed literature, we would like to point out several possible pitfalls when incorporating skills in workforce planning models. One of the most important requirements for a successful implementation of skills in a workforce planning problem is the correct classification of the skills that are used. Since categorical skills give rise to other consequences than hierarchical skills when substitution or cross-training is introduced, defining the skill class is very important. Hence, research papers that lack this important definition often fail to propose a realistic and useful model.

Another pitfall is the use of bad assumptions underlying the model. We would like to warn the researcher for the missing (or the ill-considered) link between skill determinants and skill consequences. When task restrictions are the only consequence of skills in the model, it is easy to incorporate the link between the worker's licenses, qualifications or even experience and the tasks he or she is allowed to perform. When the skill consequences concern the efficiency or quality of work, the exact link between the skill determinants and consequences is less easy to establish. We only found one paper that thoroughly describes the link between the skill determinants and skill consequences. Furthermore, most of the papers we considered in this literature 
review do not give a clear description of how they determine the resulting efficiency or performance change because of substitution or cross-training. It is no use to propose a complex model when the underlying assumptions are not accurately defined. One can also not expect such a model to produce applicable solutions.

Even with correct assumptions, some models proposed in the literature still fail to represent a realistic scenario. In order to solve a realistic problem efficiently, simplifications are often needed. Therefore, some complicating elements such as preferences, decisions about the composition of working shifts and stochasticity, are often excluded from the model. Especially stochasticity appears to be a very difficult element to deal with. Despite the fact that most researchers recognize the importance and ubiquity of stochasticity, it remains the most popular topic of the future research section in many papers.

Workforce planning tends to be a very difficult problem, even without the inclusion of stochasticity. The problem is known to become even harder when skills, shifts and multiple criteria are considered. Therefore, it is almost impossible to solve problems of realistic size to optimality. This is however not always necessary because the management of a company often prefers a fast and good solution to the optimal solution. Hence, it is not surprising that researchers who are concerned with realistic problems resort to heuristic solution methods instead of exact approaches.

Finally, we found that the number of research papers presenting a model that has been applied to a real life problem is very limited. Therefore, we would like to encourage researchers to test their model on real life cases. As a final remark, we think that the workforce planning literature regarding skills would greatly benefit from research that is concerned with real life problems, proposing fast and good heuristics, using assumptions based on sound empirical evidence and does not neglect uncertainty. 
[1] U. Aickelin and K. A. Dowsland. An indirect genetic algorithm for a nurse scheduling problem. Computers $\&$ Operations Research, 31:761-778, 2004.

[2] U. Aickelin and P. White. Building better nurse scheduling algorithms. Annals of Operations Research, 128(1-4):159-177, 2004.

[3] U. Aickelin, E. K. Burke and L. Jingpeng. An evolutionary squeaky wheel optimization approach to personnel scheduling. Evolutionary Computation, IEEE Transactions on, 13(2):433 - 443, april 2009.

[4] Aiying and Rong. Monthly tour scheduling models with mixed skills considering weekend off requirements. Computers and Industrial Engineering, 59(2):334 343,2010 .

[5] H. K. Alfares. Survey, Categorization, and Comparison of Recent Tour Scheduling Literature. Annals of Operations Research, 127:145-175, 2004.

[6] L. Argote. Organizational Learning: Creating, Retaining, and Transferring Knowledge. Kluwer Academic Publishers, Norwell, MA, USA, 1st edition, 1999.

[7] A. N. Avramidis, W. Chan, M. Gendreau, P. L'Ecuyer and O. Pisacane. Optimizing daily agent scheduling in a multiskill call center. European Journal of Operational Research, 200(3):822 - 832, 2010.

[8] B. Bachelet and L. Yon. Model enhancement: Improving theoretical optimization with simulation. Simulation Modelling Practice and Theory, 15(6):703 $715,2007$.

[9] R. Bai, E. K. Burke, G. Kendall, J. Li and B. McCollum. A hybrid evolutionary approach to the nurse rostering problem. Evolutionary Computation, IEEE Transactions on, 14(4):580-590, 2010.

[10] J. F. Bard and H. W. Purnomo. A column generation-based approach to solve the preference scheduling problem for nurses with downgrading. Socio-Economic Planning Sciences, 39(3):193 - 213, 2005.

[11] J. F. Bard and H. W. Purnomo. Preference scheduling for nurses using column generation. European Journal of Operational Research, 164(2):510 - 534, 2005.

[12] J. F. Bard and L. Wan. Workforce design with movement restrictions between workstation groups. Manufacturing $\& 3$ Service Operations Management, 10(1): 24-42, January 2008.

[13] J. F. Bard, C. Binici and A. H. deSilva. Staff scheduling at the United States postal service. Computers \& Operations Research, 30(5):745 - 771, 2003.

[14] J. J. Bartholdi. A guaranteed-accuracy round-off algorithm for cyclic scheduling and set covering. Operations Research, 29(3):501-510, May/June 1981. 
[15] R. Batta, O. Berman and Q. Wang. Balancing staffing and switching costs in a service center with flexible servers. European Journal of Operational Research, 177(2):924 - 938, 2007.

[16] G. Beddoe, S. Petrovic and J. Li. A hybrid metaheuristic case-based reasoning system for nurse rostering. Journal of Scheduling, 12(2):99-119, April 2009.

[17] G. R. Beddoe and S. Petrovic. Selecting and weighting features using a genetic algorithm in a case-based reasoning approach to personnel rostering. European Journal of Operational Research, 175(2):649 - 671, 2006.

[18] J. Beliën. Exact and heuristic methodologies for scheduling in hospitals: problems, formulations and algorithms. Katholieke Universiteit Leuven, Leuven, 2006. Ph.D. dissertation.

[19] O. Bellenguez and E. Néron. Lower bounds for the multi-skill project scheduling problem with hierarchical levels of skills. In E. Burke and M. Trick, editors, Practice and Theory of Automated Timetabling V, volume 3616 of Lecture Notes in Computer Science, pages 229-243. Springer Berlin Heidelberg, 2005.

[20] O. Bellenguez-Morineau and E. Néron. A branch-and-bound method for solving multi-skill project scheduling problem. RAIRO - Operations Research, 41:155170, 2007.

[21] A. J. Benavides, M. Ritt and C. Miralles. Flow shop scheduling with heterogeneous workers. European Journal of Operational Research, 237(2):713 - 720, 2014.

[22] M. J. Bester, I. Nieuwoudt and J. H. Vuuren. Finding good nurse duty schedules: a case study. Journal of Scheduling, 10(6):387-405, December 2007.

[23] J. Bhadury, E. J. Mighty and H. Damar. Maximizing workforce diversity in project teams: A network flow approach. Omega, 28(2):143 - 153, 2000.

[24] R. Bhatnagar, V. Saddikutti and A. Rajgopalan. Contingent manpower planning in a high clock speed industry. International Journal of Production Research, 45(9):2051-2072, 2007.

[25] B. Bilgin, P. De Causmaecker, B. Rossie and G. Vanden Berghe. Local search neighbourhoods for dealing with a novel nurse rostering model. Annals of $\mathrm{Op}$ erations Research, 194(1):33-57, 2012.

[26] C. Branson, B. Badger and F. Dobbs. Patient satisfaction with skill mix in primary care: A review of the literature. Primary Health Care Research and Development, 4:329-339, 2003.

[27] P. Brucker, E. K. Burke, T. Curtois, R. Qu and G. Vanden Berghe. A shift sequence based approach for nurse scheduling and a new benchmark dataset. Journal of Heuristics, 16(4):559-573, August 2010. 
[28] J. O. Brunner, J. F. Bard and R. Kolisch. Midterm scheduling of physicians with flexible shifts using branch and price. IIE Transactions, 43(2):84-109, 2011.

[29] J. O. Brunner and G. Edenharter. Long term staff scheduling of physicians with different experience levels in hospitals using column generation. Health Care Management Science, 14(2):189-202, June 2011.

[30] J. Buchan and M. R. Dal Poz. Skill mix in the health care workforce: Reviewing the evidence. Bulletin of the World Health Organization, 80(7):575-580, 2002.

[31] E. K. Burke, P. De Causmaecker, G. Vanden Berghe and H. Van Landeghem. The state of the art of nurse rostering. Journal of Scheduling, 7(6):441-499, 2004 .

[32] E. K. Burke, P. De Causmaecker, S. Petrovic and G. Vanden Berghe. Metaheuristics for handling time interval coverage constraints in nurse scheduling. Applied Artificial Intelligence, 20(9):743-766, 2006.

[33] E. K. Burke, T. Curtois, R. Qu and G. Vanden Berghe. A scatter search methodology for the nurse rostering problem. Journal of the Operational Research Society, 61(11):1667-1679, 2010.

[34] X. Cai and K. N. Li. A genetic algorithm for scheduling staff of mixed skills under multi-criteria. European Journal of Operational Research, 125(2):359 $369,2000$.

[35] G. M. Campbell. A two-stage stochastic program for scheduling and allocating cross-trained workers. Journal of the Operational Research Society, 62(6):10381047, 2011.

[36] G. M. Campbell and M. Diaby. Development and evaluation of an assignment heuristic for allocating cross-trained workers. European Journal of Operational Research, 138(1):9 - 20, 2002.

[37] P. Cappanera and G. Gallo. A multicommodity flow approach to the crew rostering problem. Operations Research, 52(4):583-596, 2004.

[38] S. Carlos, A. Sánchez and S. Martorell. Model to study the effect of workforce on a safety equipment and its optimization. Mathematical and Computer Modelling, 54(7-8):1808 - 1812, 2011.

[39] R. C. Carrasco. Long-term staff scheduling with regular temporal distribution. Computer Methods and Programs in Biomedicine, 100(2):191-199, November 2010.

[40] S. R. Chakravarthy and S. R. Agnihothri. Optimal workforce mix in service systems with two types of customers. Production and Operations Management, 14(2):218-231, 2005. 
[41] R. Cipriano, L. Di Gaspero and A. Dovier. Hybrid approaches for rostering: A case study in the integration of constraint programming and local search. In Proceedings of the Third international conference on Hybrid Metaheuristics, HM06, pages 110-123, Berlin, Heidelberg, 2006. Springer-Verlag.

[42] A. Corominas, R. Pastor and J. Plans. Balancing assembly line with skilled and unskilled workers. Omega, 36(6):1126 - 1132, 2008.

[43] A. Corominas, J. Olivella and R. Pastor. Capacity planning with working time accounts in services. Journal of the Operational Research Society, 61(2):321$331,2010$.

[44] A. Corominas, A. Lusa and J. Olivella. A detailed workforce planning model including non-linear dependence of capacity on the size of the staff and cash management. European Journal of Operational Research, 216(2):445 - 458, 2012 .

[45] F. Crossan and D. Ferguson. Exploring nursing skill mix: A review. Journal of Nursing Management, 13:356-362, 2005.

[46] V. Currie, G. Harvey, E. West, H. McKenna and S. Keeney. Relationship between quality of care, staffing levels, skill mix and nurse autonomy: literature review. Journal of Advanced Nursing, 51(1):73-82, 2005.

[47] R. de Matta and E. Peters. Developing work schedules for an inter-city transit system with multiple driver types and fleet types. European Journal of Operational Research, 192(3):852 - 865, 2009.

[48] B. Detienne, L. Péridy, E. Pinson and D. Rivreau. Cut generation for an employee timetabling problem. European Journal of Operational Research, 197(3): $1178-1184,2009$.

[49] L. E. Drezet and J. C. Billaut. A project scheduling problem with labour constraints and time-dependent activities requirements. International Journal of Production Economics, 112(1):217 - 225, 2008.

[50] C. du Boulay and C. Medway. The clinical skills resource: A review of current practice. Medical Education, 33:185-191, 1999.

[51] H. A. Eiselt and V. Marianov. Employee positioning and workload allocation. Computers $\& 3$ Operations Research, 35(2):513 - 524, 2008.

[52] G. Eitzen, D. Panton and G. Mills. Multi-skilled workforce optimisation. Annals of Operations Research, 127:359-372, 2004.

[53] A. T. Ernst, H. Jiang, M. Krishnamoorthy and D. Sier. Staff scheduling and rostering: A review of applications, methods and models. European Journal of Operational Research, 153:3-27, 2004. 
[54] K. Ertogral and B. Bamuqabel. Developing staff schedules for a bilingual telecommunication call center with flexible workers. Computers $\&$ Industrial Engineering, 54(1):118 - 127, 2008.

[55] P. Eveborn, P. Flisberg and M. Rönnqvist. Laps Care - An operational system for staff planning of home care. European Journal of Operational Research, 171 (3):962 - 976, 2006.

[56] M. Firat and C. A. J. Hurkens. An improved mip-based approach for a multiskill workforce scheduling problem. Journal of Scheduling, 15:363-380, 2012.

[57] J. W. Fowler, P. Wirojanagud and E. S. Gel. Heuristics for workforce planning with worker differences. European Journal of Operational Research, 190(3):724 $-740,2008$.

[58] E. Fragnière, J. Gondzio and X. Yang. Operations risk management by optimally planning the qualified workforce capacity. European Journal of Operational Research, 202(2):518 - 527, 2010.

[59] M. François. Symmetry in Integer Linear Programming. 50 Years of Integer Programming, Springer, Carnegie Mellon University, 2009.

[60] I. P. Gent, K. E. Petrie and J.-F. Puget. Chapter 10: Symmetry in constraint programming. In Handbook of Constraint Programming, volume 2 of Foundations of Artificial Intelligence, pages 329-376. Elsevier, 2006.

[61] I. Gibbs, D. McCaughan and M. Griffiths. Skill mix in nursing: A selective review of the literature. Journal of Advanced Nursing, 16:242-249, 1991.

[62] F. Glover and C. McMillan. The general employee scheduling problem: An integration of MS and AI. Computers and Operations Research, 13(5):563-573, 1986.

[63] M. Golalikhani and M. H. Karwan. A hierarchical procedure for multi-skilled sales force spatial planning. Computers \& Operations Research, 40(5):1467 $1480,2013$.

[64] M. D. Goodman, K. A. Dowsland and J. M. Thompson. A grasp-knapsack hybrid for a nurse-scheduling problem. Journal of Heuristics, 15(4):351 - 379, 2009 .

[65] L. Gordon and E. Erkut. Improving volunteer scheduling for the edmonton folk festival. Interfaces, 34(5):367 - 376, 2004.

[66] M. Günther and V. Nissen. Particle swarm optimization and an agent-based algorithm for a problem of staff scheduling. In Applications of Evolutionary Computation, volume 6025 of Lecture Notes in Computer Science, pages 451 461. Springer Berlin Heidelberg, 2010. 
[67] W. J. Gutjahr and M. S. Rauner. An ACO algorithm for a dynamic regional nurse-scheduling problem in Austria. Computers $\& 3$ Operations Research, 34(3): $642-666,2007$.

[68] T. Hanne, R. Dornberger and L. Frey. Multiobjective and preference-based decision support for rail crew rostering. In Evolutionary Computation, 2009. CEC 'O9. IEEE Congress on, pages 990 - 996, 2009.

[69] P. R. Harper, N. H. Powell and J. E. Williams. Modelling the size and skill-mix of hospital nursing teams. Journal of the Operational Research Society, 61(5): 768-779, May 2010.

[70] C. Heimerl and R. Kolisch. Scheduling and staffing multiple projects with a multi-skilled workforce. OR Spectrum, 32(2):343 - 368, 2010.

[71] S. Helber and K. Henken. Profit-oriented shift scheduling of inbound contact centers with skills-based routing, impatient customers, and retrials. OR Spectrum, 32(1):109-134, 2010.

[72] S. C. Ho and J. M. Y. Leung. Solving a manpower scheduling problem for airline catering using metaheuristics. European Journal of Operational Research, 202 (3):903 - 921, 2010 .

[73] M. Hojati. Near-optimal solution to an employee assignment problem with seniority. Annals of Operations Research, 181(1):539 - 557, 2010.

[74] M. Hojati and A. S. Patil. An integer linear programming-based heuristic for scheduling heterogeneous, part-time service employees. European Journal of Operational Research, 209(1):37 - 50, 2011.

[75] W. J. Hopp and M. P. Oyen. Agile workforce evaluation: A framework for cross-training and coordination. IIE Transactions, 36(10):919-940, 2004.

[76] H.-C. Huang, L.-H. Lee, H. Song and B. T. Eck. SimMan - A simulation model for workforce capacity planning. Computers $\& 3$ Operations Research, 36(8):2490 - 2497, 2009.

[77] J. E. Hunter. Cognitive ability, cognitive aptitudes, job knowledge, and job performance. Journal of Vocational Behavior, 29(3):340 - 362, 1986.

[78] A. Janiak and R. Rudek. A note on a makespan minimization problem with a multi-ability learning effect. Omega, 38(3-4):213 - 217, 2010.

[79] K. H. Kim, K. W. Kim, H. Hwang and C. S. Ko. Operator-scheduling using a constraint satisfaction technique in port container terminals. Computers 83 Industrial Engineering, 46(2):373 - 381, 2004.

[80] S. Knust and E. Schumacher. Shift scheduling for tank trucks. Omega, 39(5): $513-521,2011$. 
[81] N. Kohl and S. E. Karisch. Airline crew rostering: Problem types, modeling, and optimization. Annals of Operations Research, 127(1-4):223-257, 2004.

[82] M. Krishnamoorthy, A. T. Ernst and D. Baatar. Algorithms for large scale shift minimisation personnel task scheduling problems. European Journal of Operational Research, 2011.

[83] A. G. Lagodimos and V. Leopoulos. Greedy heuristic algorithms for manpower shift planning. International Journal of Production Economics, 68(1):95 - 106, 2000 .

[84] A. G. Lagodimos and A. N. Mihiotis. Overtime vs. regular shift planning decisions in packing shops. International Journal of Production Economics, 101(2): $246-258,2006$.

[85] W.-C. Lee and C.-C. Wu. A note on single-machine group scheduling problems with position-based learning effect. Applied Mathematical Modelling, 33(4):2159 $-2163,2009$.

[86] Y. S. Lee. The effect of workers with different capabilities on customer delay. Computers $\& 3$ Operations Research, 31(3):359 - 381, 2004.

[87] J. Li, U. Aickelin and E. K. Burke. A component-based heuristic search method with evolutionary eliminations for hospital personnel scheduling. INFORMS Journal on Computing, 21(3):468-479, July 2009.

[88] N. Li and L. X. Li. Modeling staffing flexibility: A case of China. European Journal of Operational Research, 124(2):255 - 266, 2000.

[89] L. Liberti. Mixed Integer Nonlinear Programming, chapter Symmetry in Mathematical Programming, 263-283. Springer New York, LIX, École Polytechnique, 2012.

[90] B. Maenhout and M. Vanhoucke. A hybrid scatter search heuristic for personalized crew rostering in the airline industry. European Journal of Operational Research, 206(1):155 - 167, 2010.

[91] B. Maenhout and M. Vanhoucke. An integrated nurse staffing and scheduling analysis for longer-term nursing staff allocation problems. Omega, 41(2):485 499, 2013.

[92] T. Majozi and X. X. Zhu. A combined fuzzy set theory and milp approach in integration of planning and scheduling of batch plants-personnel evaluation and allocation. Computers 83 Chemical Engineering, 29(9):2029 - 2047, 2005.

[93] K. A. Marentette, A. W. Johnson and L. Mills. A measure of cross-training benefit versus job skill specialization. Computers $\mathcal{E}$ Industrial Engineering, 57 (3):937 - 940, 2009.

[94] I. Markes. A review of literature on employability skill needs in engineering. European Journal of Engineering Education, 31(6):637-650, 2006. 
[95] D. A. Nembhard and B. A. Norman. Worker efficiency and responsiveness in cross-trained teams. Technical Report, University of Wisconsin, Madison, 2002.

[96] R. Pastor and A. Corominas. A bicriteria integer programming model for the hierarchical workforce scheduling problem. Journal of modelling in management, $5(1): 54-62,2010$.

[97] E. J. Pinker and R. A. Shumsky. The efficiency-quality trade-off of cross-trained workers. Manufacturing $\&$ Service Operations Management, 2(1):32-48, January 2000 .

[98] S. J. Sadjadi, R. Soltani, M. Izadkhah, F. Saberian and M. Darayi. A new nonlinear stochastic staff scheduling model. Scientia Iranica, 18(3):699 - 710, 2011.

[99] N. Safaei, D. Banjevic and A. K. S. Jardine. Workforce-constrained maintenance scheduling for military aircraft fleet: a case study. Annals of Operations Research, 186(1):295-316, 2011.

[100] S. Sayin and S. Karabati. Assigning cross-trained workers to departments: A two-stage optimization model to maximize utility and skill improvement. European Journal of Operational Research, 176(3):1643 - 1658, 2007.

[101] E. Scott and Sampson. Optimization of volunteer labor assignments. Journal of Operations Management, 24(4):363 - 377, 2006.

[102] S. U. Seçkiner, H. Gökçen and M. Kurt. An integer programming model for hierarchical workforce scheduling problem. European Journal of Operational Research, 183(2):694-699, 2007.

[103] P. Smet, B. Bilgin, P. De Causmaecker and G. Vanden Berghe. Modelling and evaluation issues in nurse rostering. Annals of Operations Research, pages 1-24, 2012 .

[104] H. Song and H.-C. Huang. A successive convex approximation method for multistage workforce capacity planning problem with turnover. European Journal of Operational Research, 188(1):29 - 48, 2008.

[105] J. K. Stratman, A. V. Roth and W. G. Gilland. The deployment of temporary production workers in assembly operations: A case study of the hidden costs of learning and forgetting. Journal of Operations Management, 21(6):689 - 707, 2004 .

[106] A. Techawiboonwong, P. Yenradee and S. K. Das. A master scheduling model with skilled and unskilled temporary workers. International Journal of Production Economics, 103(2):798 - 809, 2006.

[107] G. M. Thompson and J. C. Goodale. Variable employee productivity in workforce scheduling. European Journal of Operational Research, 170(2):376 - 390, 2006. 
[108] V. Tiwari, J. H. Patterson and V. A. Mabert. Scheduling projects with heterogeneous resources to meet time and quality objectives. European Journal of Operational Research, 193(3):780 - 790, 2009.

[109] E. Tsang and C. Voudouris. Fast local search and guided local search and their application to British Telecom's workforce scheduling problem. Operations Research Letters, 20(3):119 - 127, 1997.

[110] V. Valls, A. Pérez and S. Quintanilla. Skilled workforce scheduling in service centres. European Journal of Operational Research, 193(3):791 - 804, 2009.

[111] J. Van den Bergh, J. Beliën, P. De Bruecker, E. Demeulemeester and L. De Boeck. Personnel scheduling: A literature review. European Journal of Operational Research, 226(3):367 - 385, 2013.

[112] P. H. Vance, C. Barnhart, E. L. Johnson and G. L. Nemhauser. Airline crew scheduling: A new formulation and decomposition algorithm. Operations Research, 45(2):188-200, 1997.

[113] R. B. Wallace and W. Whitt. A staffing algorithm for call centers with skillbased routing. Manufacturing \& Service Operations Management, 7(4):276-294, 2005 .

[114] N. Wongwai and S. Malaikrisanachalee. Augmented heuristic algorithm for multi-skilled resource scheduling. Automation in Construction, 20(4):429 - 445, 2011.

[115] C.-C. Wu and W.-C. Lee. Single-machine scheduling problems with a learning effect. Applied Mathematical Modelling, 32(7):1191 - 1197, 2008.

[116] C.-C. Wu, P.-H. Hsu and K. Lai. Simulated-annealing heuristics for the singlemachine scheduling problem with learning and unequal job release times. Journal of Manufacturing Systems, 30(1):54 - 62, 2011.

[117] C.-C. Wu, S.-K. Huang and W.-C. Lee. Two-agent scheduling with learning consideration. Computers \& Industrial Engineering, 61(4):1324 - 1335, 2011.

[118] Z. Xingong and Y. Guangle. Single-machine group scheduling problems with deteriorated and learning effect. Applied Mathematics and Computation, 216 (4):1259 - 1266, 2010.

[119] L. E. Yelle. The learning curve: Historical review and comprehensive survey. Decision Sciences, 10(2):302-328, 1979.

[120] G. Zülch, S. Rottinger and T. Vollstedt. A simulation approach for planning and re-assigning of personnel in manufacturing. International Journal of Production Economics, 90(2):265 - 277, 2004. 